\title{
QUANTITATIVE CRITERION OF INDIVIDUAL DIFFERENCES OF THE EYES MOVEMENT TRAJECTORIES
}

\author{
Rostislav V. Belyaev, Vladimir V. Kolesov \\ Kotel'nikov Institute of Radio-Engineering and Electronics of RAS, http://www.cplire.ru \\ 125009 Moscow, Russian Federation \\ kvv@cplire.ru,belyaev@cplire.ru
}

Galina Ya. Men'shikova

Lomonosov Moscow State University, Faculty of Psychology, http://www.psy.msu.ru,

125009 Moscow, Russian Federation

men'shikova@psy.msu.ru

\begin{abstract}
Alexander M. Popov, Viktor I. Ryabenkov
Moscow State Technical University of Radio Engineering, Electronics and Automation (MIREA), http://www.mirea.ru 119454 Moscow, Russian Federation

ryabenkov@mirea.ru,popov@mirea.ru
\end{abstract}

Abstract. It is known that eyes moving tracks recorded by special design through equal time intervals while observing images have two basic appearances - saccades and fixations. Investigation's aim was the development quantity fractal criteria of finding individual recognizable state of moving different observer's eyes. The method of the fractal analysis consists in research of characteristics of same object at different scales. Criteria evaluation's algorithm include sampling out of eyes trajectory saccades and fixation distinguished collect in adequate data sequence on $X$ and $Y$ operated separately. Every realization is operated through measuring window on z-steps data access in time sliding along realization by single step. On every step there were defined separately for realization saccades and fixation standard deviation $\sigma \mathrm{x}$ on $\mathrm{X}$ and $\sigma \mathrm{y}$ on $\mathrm{Y}$ and computed adequate $\sigma(\mathrm{i})$ on full window size as $\sigma(\mathbf{i})=\sigma(x)=\sqrt{\sigma_{x}^{2}(i+j)+\sigma_{y}^{2}(i+j)}$, where $\mathbf{i}=1:(\mathbf{N}-\mathbf{z}+\mathbf{1})$ - number shift's step on realization length- $N$ and $j=1: z$ on full windows length. The measuring window's size was changed as $z=1: z_{\text {max }}$. For every observer was performed data smoothing for images turn on with step-angle $45^{\circ}\left(0^{\circ}-315^{\circ}\right)$. It was shown that smoothed by this operation values $\sigma_{\text {mean }}(z)$ formed linear functional dependence $\mathrm{y}=\mathrm{a}+\mathrm{bx}$ with substantially different values of coefficients a and $b$ for variety observers for saccades and fixations that provide individual observers.

Keywords: information technologies, cognitive processes technologies, eyes movements, perception of video information, psychophysiological features of visual organs, fractal analysis, fractal dimension.

UDC 004.932.2; 159.931

Bibliograpby -4 references

Received 20.05.2014

RENSIT, 2015, 7(1):25-33

DOI: $10.17725 /$ rensit.2015.07.025

\section{Contents}

\section{IntRoduction (25)}

2. Equipment (26)

3. Description of the Method (27)

4. Method of fractal processing (29)

5. Conclusion (32)

REFERENCES (33)

\section{INTRODUCTION}

Eye movements perform a variety of functions in creation of a visual image and space perception, allowing an estimation of dimension and analysis of spatial properties for observed objects.

The main physiological characteristics, which can be used to assess different cognitive processes, are such characteristics as a number 
and duration of fixations, saccade speed and direction, regressive eye movements. It is shown that the specified parameters can successfully explain regularities of processes of a visual field search, reading, decisionmaking and many others. However, these traditionally measured characteristics are too simplified for an estimation of complex cognitive processes. Attempts to find more adequate processing forms for patterns of eye movements, which will allow to provide both distinction of observers and observation objects, are made. Traditionally, researches of eye movements have been studied by various methods of statistics and clearly show the differences in statistics for different observers and objects, but it is advisable to find quantitative methods to obtain relevant comparative estimations.

\section{EQUIPMENT}

Eye movements were registered using a computer device iV iew XTM Hi-Speed 1250 of the German company "SMI GmbH" (resolution $<0.01^{\circ}$, data sampling frequency $1250 \mathrm{~Hz}$ ). It provided a non-contact method of determining a location of an observer's eye on monitor screen, where an observation object's image, called "dark pupil system", was found. According to it, an observer's eye was lit by an infrared radiation (IR), which is well reflected by all areas of an eye surface, except for a pupil area.

Parameters of the eye IR-image were registered by a video camera and were recorded in a data file. Sampling of each measurement result was performed through an interval of $20 \mathrm{~ms}(500 \mathrm{~Hz})$ or $8 \mathrm{~ms}$ $(1250 \mathrm{~Hz})$ and coordinates were recorded in pixels of a pupil center (in the camera coordinate system), horizontal and vertical pupil "diameter" in the perspective eye
IR-image. Prior to the main experiment, a mandatory calibration procedure (the socalled "validation") was conducted, according to which the task to look one-by-one at some test reference points on monitor screen was set for a test person. The test person's head was fixed. The distance from the test person's eyes to the monitor screen was equal to $75 \mathrm{~cm}$. The screen size was $32 \times 24 \mathrm{~cm}$ and $24 \times 16$ arc degrees in angular units.

Among several possible types of eye movements, which vary in time and space characteristics, 3 main characteristics of eye movements were recorded in the device the object's observation. It includes: saccades, fixations and eye movement patterns while observing images on monitor screen, which is formed in successive samplings from fixations and saccades and contains information on strategy of information gathering when performing certain set tasks. Display trajectories of eye movement are formed by connecting the points with coordinates, determined consistently in time, in the course of periodic samplings at regular time intervals ( $\mathrm{T}=0.002 \mathrm{~s}$ or $0.0008 \mathrm{~s})$.

Typical result type of data registration for shift of a person's look on a flat image's surface for the example of such movements is shown in Fig. 1. Here is an example of

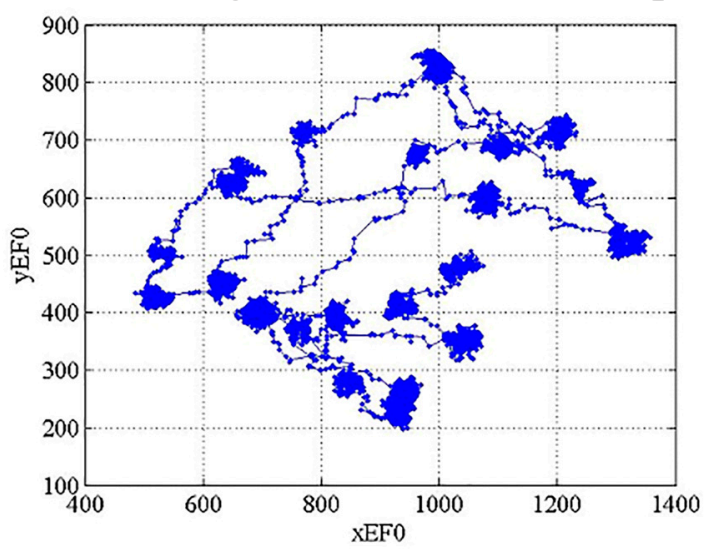

Fig. 1. Realization patterns of eyes movements without division on saccades and fixations while observing some image $x E F O-y E F O$. There are clearly seen local regions of fixations and well-defined saccadic movements. Points on curves mark the moments of holding samples. 
an eye movement's realization (pattern) without separation of saccades and fixations. Points on curves correspond to sampling moments - registration of an instantaneous position of an eye pupil center. Time interval between two consecutive sampling points is constant and determined by the used sampling frequency. Areas of concentration of sampling points' position correspond to fixations (microsaccades) when eye moving, and more or less equal areas with small deviations and clearly visible sampling moments that correspond to saccades. Shifts in fixations on average have lower values than in area of saccades. Besides, they differ in a sharp change of the point's shift direction, displaying successive selection moments. These angles between shift directions can make a value close to $2 \pi$.

It should be noted that in the process of an image's monitoring, reflected in a pattern, the total stay period of an observer's look in areas of fixations usually significantly (by times) exceeds the total time, spent in shifts, defined as a saccade.

The work objective is a search for methods of distinguishing individual observers based on specifics of movement characteristics of their eyes when looking at the monitor screen using multi-scale fractal approach. It is performed when a movement realization of an observer's eye when viewing an image is visible sequentially through an averaging window, moving along its length, which size then sequentially changed by one step during realization.

In the course of work with use of the above equipment, an observer's eye movement during viewing of an motionless flat image were registered sequentially. Thus, the coordinates $(X(i), Y(i))$ of instant positions of an observer's look with fixed sampling frequency were memorized in a field of the selected image, recalculated on change in time of an eye pupil's position [1].

\section{DESCRIPTION OF THE METHOD}

As the eye movements in areas of fixations and saccades are different in their spatial and temporal characteristics, so the first stage of the work is their allocation from the registered movement realization for a subsequent separate processing using the developed algorithm. This separation was performed in accordance with separation criteria, details of which are given in other works of the authors [2]. Such separation can also be performed automatically using an approach, put in the equipment program.

The separation criteria can be a nature of current changes in spatiotemporal characteristics of sequence of the registered realization. For example, sharp change of movement speed for an interval between two or several consecutive time selections. The assessment also can be made based on sharpness of change of a movement direction.

For formation of a corresponding data file, each revealed realization area, relating to one of two movement types (saccade and fixation), was filed to the corresponding end of the previously selected area with the same movement character [3].

Thus, the whole registered realization was processed. Measuring equipment fixes values of the $X(i)$ and $Y(i)$ coordinates, displaying an instant position of a representing point of an observer's look at a given time. As a result, two realizations, corresponding to sequences 
with this movement type on coordinates of $X$ and $Y$, turns out.

Each realization was divided into saccade movement and fixation movement and was processed with use of a measuring window with a variable number of $z$-steps in samplings on time, sliding along the whole realization with a single step. Mean square deviations of $\sigma_{\mathrm{x}}$ in $X$ and $\sigma_{\mathrm{y}}$ in $Y$ were determined separately on each step for saccade movement and fixation realization and the corresponding values of $\sigma(\imath)$ were calculated throughout the window's size $\sigma(x)=\sqrt{\sigma_{x}^{2}(i+j)+\sigma_{y}^{2}(i+j)}$, where $i=1:(N$ $-z+1)-$ number of the window's shift step in realization with length $N$, and $j=$ 1:z changes based on the window's width. Size of a measuring window varies as $z$ $=1: z_{\max }$ A window with a size $z=1$ on each $i$ step on a sequence length $i=1: \mathrm{N}$ contains only one next realization member and formally corresponding values of $\sigma(i)$ for all $i$, equal to 0 . This value is left in creation of corresponding graphs in figures for presentation clarity of further course of displaying sequences. Then, averaging of values $\sigma(\imath)$ according to 8 images, received by a turn of one image with a step by an angle of $45^{\circ}$ in the range from $0^{\circ}$ to $315^{\circ}$ was made for each observer.

During experiments, each observer was offered for viewing 3 different images, which are conditionally were called: fractal $(F)$, tree $(T)$ and wave $(W)$. The full data volume on samplings for each observer made: for one image and full length of realization $\sim 10000$ 12000 , from which about a tenth $\sim 1000$ 1200 were saccades, and the rest - fixations 2000-10800. Viewing of 24 images was carried out for 3 images (and 8 turns for each of them). Thus, the total volume of samplings during realization for one observer was: $240000-288000$.

The window $z=1$ formally has a size of 0 (zero) and is used for convenience of construction of charts and assessment of coefficients, approximating the course of linear relations.

The first real measuring window $z=$ 2 corresponds to one real step, that is a distance between two successive readings of realization and, thus, it is possible to estimate sizes of a mean square step of realizations for each observer separately for saccade movement and fixations. All subsequent assessments $(z>2)$ give the results, related to size of an averaging window, sliding in the course of realization. Thus, an average definition of a specified $\sigma(i)$ is made for this size of a window on each step.

Assessments for a random process, formed by a random number generator separately for coordinates of $X$ and $Y$ in an assumption that dispersions of processes on $X$ and $Y$ are equal against each other and equal to 1 , are also carried out for comparison.

It was found that the average $\sigma_{\text {mean }}(z)$ in realizations have a rather long section with functional dependence, close to linear $y=$ $a+b x$, but with slightly different values of the coefficients $a$ and $b$ for different observers for saccades and fixations that potentially provides individual distinction of observers.

We will note that the number of ordinate values (samplings) of $X$ and $Y$, registered by equipment (after averaging by 8 successive turns of this image by an angle of $45^{\circ}$ ) makes $\sim 10^{4}-1.2 \cdot 10^{4}$ for fixations, and $\sim 10^{3}-1.2 \cdot 10^{3}$ for saccade movements (with a frequency of samplings $F=1250 \mathrm{~Hz}$ ). 


\section{METHOD OF FRACTAL PROCESSING}

We used an approach, where a viewing of registered realizations of the observer's eye movements through a window of $z$ size with averaging on each following step was performed consistently in the sliding mode [4]. Thus, we carried out a processing and subsequent averaging on a volume, equal to the window's length. This operation was performed for windows of different (increasing) length.

In all figures the calculated curves, according to the results of experiments with the image - fractal $(F)$ are carried out in the form of a continuous line, with the image tree $(T)$ as a dotted line, and with the image - wave $(W)$ in the form of dot-dash lines; and in this case a color of the line may be different for different images.

The first letters $(V, O, E$ and $Z$ ) designate an observer, small letters $s$ (or $f$ ) designate that data belong to realization, created respectively, from sequence of saccade movements $(s)$ or fixations $(f)$. The small letter $m$ indicates averaging on data for 8 turns of an image by an angle of $45^{\circ}$ (in the range of angles from $0^{\circ}$ to $315^{\circ}$ ).

If there is not only one letter $F, T$ or $W$ near an arrow, but all together, it means that dependences on graphics are almost difficult to distinguish and they are designated by a continuous line.

All calculations were made for a linear ratio $y=a+b x$, coefficients were determined with use of two extreme points of an area with a linear dependence. They are designated for each figure in Table as (X1, Y1)-(X2, Y2).

Calculations were carried out with use of two formulas, deduced in assumption of a linear character of the given dependences, which are expressed by formulas

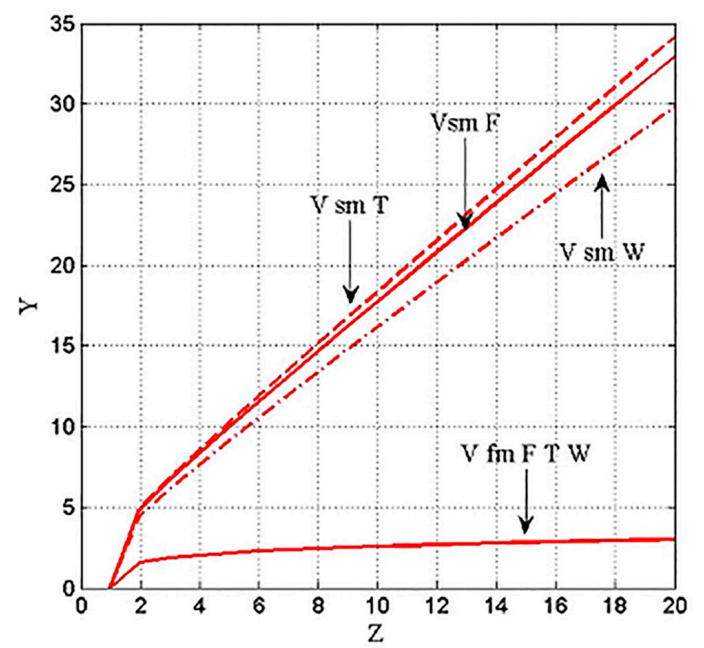

Fig. 2. Observer $V$.

Table 1 to Fig. 2.

\begin{tabular}{|c|c|c|c|c|c|c|c|}
\hline $\mathrm{V}$ & & $\mathrm{X} 1$ & $\mathrm{Y} 1$ & $\mathrm{X} 2$ & $\mathrm{Y} 2$ & $\mathrm{a}$ & $\mathrm{b}$ \\
\hline for $\mathrm{s}$ & $\mathrm{F}$ & 6 & 11.53 & 20 & 32.93 & 1.528 & 2.47 \\
\hline & $\mathrm{T}$ & 6 & 11.9 & 20 & 34.14 & 1.588 & 2.64 \\
\hline & $\mathrm{W}$ & 6 & 10.53 & 20 & 29.83 & 1.378 & 2.27 \\
\hline for $\mathrm{f}$ & $\mathrm{F}$ & 6 & 2.276 & 20 & 3.005 & 0.052 & 1.965 \\
\hline & $\mathrm{T}$ & 6 & 2.276 & 20 & 3.005 & 0.052 & 1.965 \\
\hline & $\mathrm{W}$ & 6 & 2.276 & 20 & 3.005 & 0.052 & 1.965 \\
\hline
\end{tabular}

$Y 1=a+b X 1$

$Y 2=a+b X 2$,

that are used respectively for calculation of two necessary coefficients for definition of the corresponding approximate straight lines of an inclination for dependences.

Hence, according to the results, shown in the figures, we can determine appropriate value pairs at the ends of approximately linear sections (X1, Y1) and (X2, Y2) and, accordingly, to calculate coefficients of the approximating lines $b=(Y 2-Y 1) /(X 2-$ $X 1)$ and $a=Y 2-X 2 b$.

Results of calculations for 3 observers $(V, O$ and $E$ ), each of whom looked through all 24 images, are presented in fig. 2-4. Each figure shows at the same time the results of processing by the described method for saccade movements, and for area of fixations. It is clearly seen that they are very different from each other on nature of change with growth of the window's size and averaging. The relevant data and results 


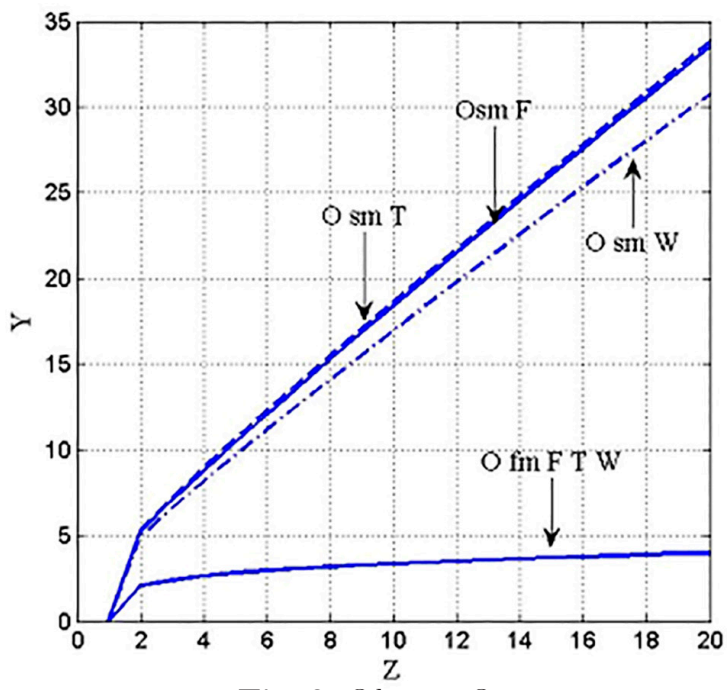

Fig. 3. Observer $O$.

Table 2 to Fig. 3.

\begin{tabular}{|c|c|c|c|c|c|c|c|}
\hline $\mathrm{O}$ & & $\mathrm{X} 1$ & $\mathrm{Y} 1$ & $\mathrm{X} 2$ & $\mathrm{Y} 2$ & $\mathrm{a}$ & $\mathrm{b}$ \\
\hline for $\mathrm{s}$ & $\mathrm{F}$ & 6 & 12.13 & 20 & 33.84 & 1.550 & 2.84 \\
\hline & $\mathrm{T}$ & 6 & 12.13 & 20 & 33.84 & 1.550 & 2.84 \\
\hline & $\mathrm{W}$ & 6 & 11.15 & 20 & 30.74 & 1.400 & 2.81 \\
\hline for $\mathrm{f}$ & $\mathrm{F}, \mathrm{T}, \mathrm{W}$ & 6 & 2.927 & 20 & 4.009 & 0.077 & 2.469 \\
\hline
\end{tabular}

of calculations for each observer are shown in Tables.

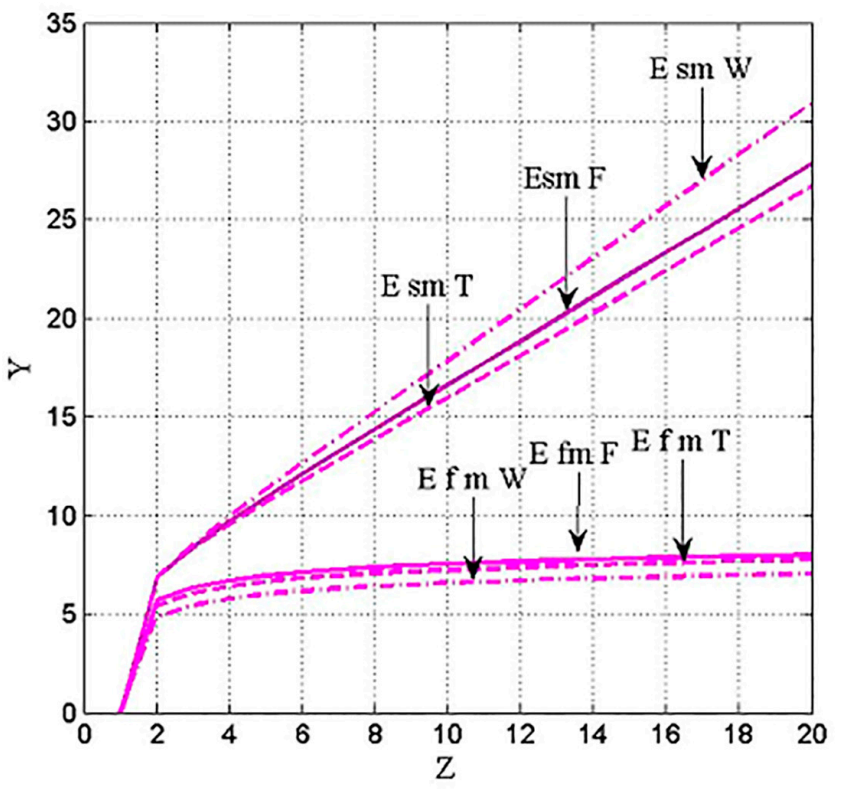

Fig. 4. Observer E.

Table 3 to Fig. 4.

\begin{tabular}{|c|c|c|c|c|c|c|c|}
\hline $\mathrm{E}$ & & $\mathrm{X} 1$ & $\mathrm{Y} 1$ & $\mathrm{X} 2$ & $\mathrm{Y} 2$ & $\mathrm{a}$ & $\mathrm{b}$ \\
\hline for s & $\mathrm{F}$ & 6 & 12.09 & 20 & 27.84 & 1.125 & 5.34 \\
\hline & $\mathrm{T}$ & 6 & 11.72 & 20 & 26.67 & 1.068 & 5.313 \\
\hline & $\mathrm{W}$ & 6 & 12.64 & 20 & 30.94 & 1.307 & 4.811 \\
\hline for f & $\mathrm{F}$ & 6 & 7.121 & 20 & 8.017 & 0.064 & 6.737 \\
\hline & $\mathrm{T}$ & 6 & 6.818 & 20 & 7.751 & 0.067 & 6.411 \\
\hline & $\mathrm{W}$ & 6 & 6.173 & 20 & 7.034 & 0.062 & 5.904 \\
\hline
\end{tabular}

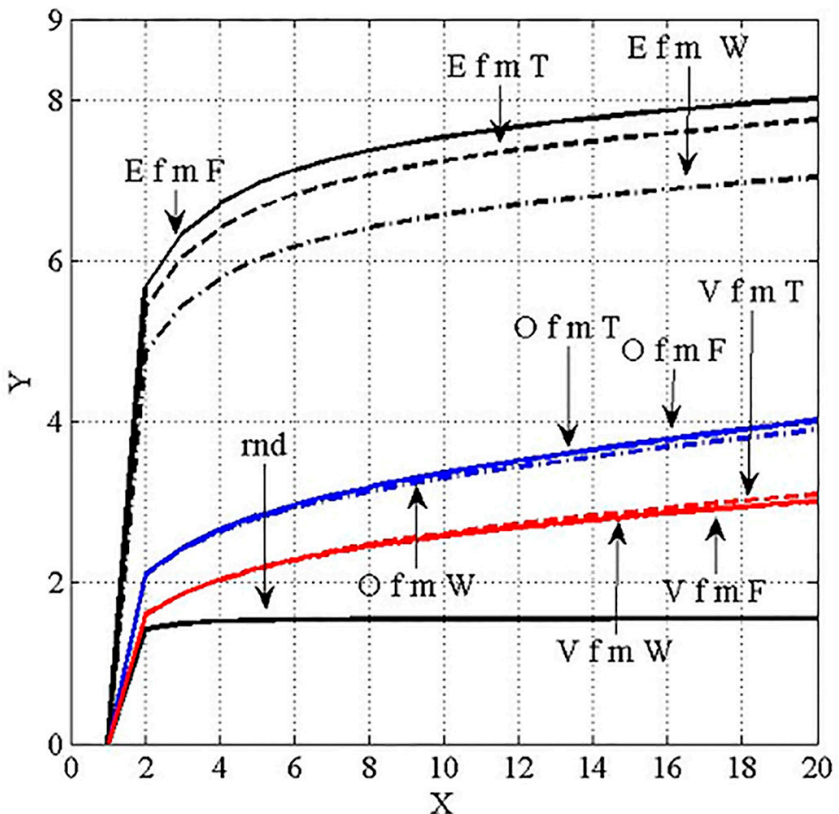

Fig. 5. Results of treating region of fixations for 3 observers ( $V$, $O, E$ ) and random process through sliding windows (with following increasing the size of windows Z) and for realization formed by generator of random numbers.

Table 4 to Fig.5.

\begin{tabular}{|c|c|c|c|c|c|c|c|}
\hline E & & $\mathrm{X} 1$ & $\mathrm{Y} 1$ & $\mathrm{X} 2$ & $\mathrm{Y} 2$ & $\mathrm{a}$ & $\mathrm{b}$ \\
\hline V-for f & $\mathrm{F}$ & 8 & 2.444 & 20 & 3.005 & 0.0467 & 2.07 \\
\hline & $\mathrm{T}$ & 8 & 2.472 & 20 & 3.096 & 0.052 & 2.056 \\
\hline & $\mathrm{W}$ & 8 & 2.444 & 20 & 3.005 & 0.0467 & 2.07 \\
\hline E-for f & $\mathrm{F}$ & 8 & 7.367 & 20 & 8.017 & 0.0541 & 6.669 \\
\hline & $\mathrm{T}$ & 8 & 7.065 & 20 & 7.751 & 0.0572 & 6.607 \\
\hline & $\mathrm{W}$ & 8 & 6.409 & 20 & 7.034 & 0.052 & 5.994 \\
\hline O- for & $\mathrm{F}$ & 8 & 3.175 & 20 & 4.009 & 0.0695 & 2.619 \\
\hline & $\mathrm{T}$ & 8 & 2.472 & 20 & 3.096 & 0.052 & 1.965 \\
\hline & $\mathrm{W}$ & 8 & 2.444 & 20 & 3.005 & 0.047 & 2.07 \\
\hline rnd & Nrnd=10000 & 8 & 1.543 & 20 & 1.554 & $9 \cdot 10^{-4}$ & 1.55 \\
\hline
\end{tabular}

For comparison, the Fig. $\mathbf{5}$ shows results of an analysis of fixations' realizations $(f)$, received with a sampling frequency of 1250 $\mathrm{Hz}$ for images using an averaging window for 3 observers $(V, O$, and $E$ ) only for fixations $(f)$ when viewing 3 images $(F, T, W)$ with averaging $(m)$ by 8 turns by an angle of $45^{\circ}$. It is evident that the viewing results for the observer $V$ through a sliding window by the method for fixations are very close to all images $F, T, W$.

We can note that the processing result by the proposed realization method of a random process, generated by a random number generator $(r n d)$, significantly differs 
from the course of other curves. First of all, the corresponding dependence for rnd very quickly comes to a small inclination with respect to the axis $X=Z$, corresponding to size of an averaging window.

Results for fixations of the observers $V$ and $O$ are very close in their values. At the same time, the corresponding characteristics for the observer $E$ on an inclination (coefficient $b$ ) are close, but significantly differ on a coefficient $a$ (approximately by 3 times more in value).

We can note that the corresponding dependences for fixations of the observer $E$ when viewing 3 images $(F, T W)$ with 8 turns by an angle $(m)$ for each one, curves are very clearly separated from each other (i.e. have a different value of the coefficient $-a$ ), but have very close values of an inclination concerning the axis $X$ (coefficient $-b)$.

Contrary to it, the curves of fixations' processing for each of observers $F, T$ and $W$ are clearly differ from each other (coefficient $-b)$.

Dependence for the rnd process, corresponding to processing by the used viewing method through a sliding window with averaging during realization, received in the course of operation of a random number generator, is given in this Fig. 5 for comparison. As we can see, this curve has extremely small inclination concerning axis $X$, so the graphic editor approximates it pieces of straight lines with the increasing length. Its course, even with small sizes of an averaging window $Z$ (represented on the axis $X$ ) practically very quickly turns into a continuous line. That is, in fact, this dependence becomes almost independent of the size value of an averaging window $Z$. It testifies that a random process (formed by a generator $r n d$ ) is characterized by rapid (within a few steps of the value of the $Z$ window's width) exit to a linear dependence.

Data on calculations for methods of the rnd realization are provided in the last row of Tab. 4. Dependences for observers $O$ and $V$ are very close by an inclination $(b)$ and coefficient $a$ (for all 3 images $-F, T$ and $W$ ).

Based on this, it can be assumed that fixation areas, apparently, differ in character from random process, i.e. they are not just noise random movements, but, perhaps, can carry out the retention function of the most sensitive part of an eye apparatus in an image's area of interest to an observer.

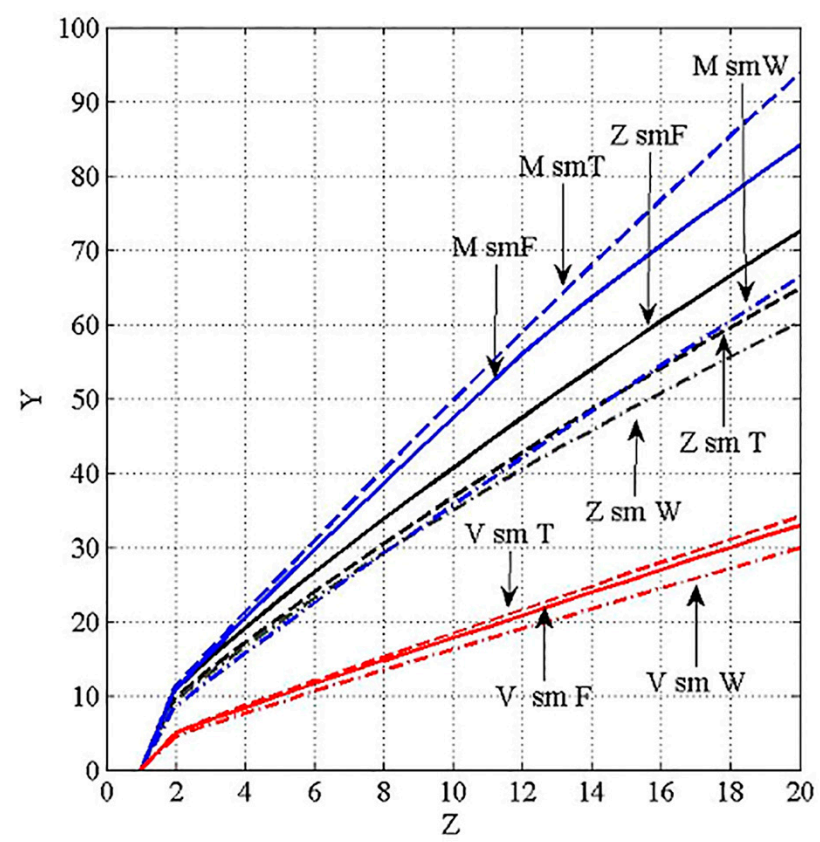

Fig. 6. Dependence for RND saccadic movements for observers $M, Z$ and $V$.

Таблича 5 крис. 6.

\begin{tabular}{|l|l|l|l|l|l|l|}
\hline For s & $\mathrm{X} 1$ & $\mathrm{Y} 1$ & $\mathrm{X} 2$ & $\mathrm{Y} 2$ & $\mathrm{~b}$ & $\mathrm{a}$ \\
\hline $\mathrm{MF}$ & 6 & 30.84 & 20 & 93.67 & 4.487 & 3.93 \\
\hline MT & 6 & 29.51 & 20 & 84.17 & 3.904 & 6.09 \\
\hline MW & 6 & 22.44 & 20 & 66.36 & 3.137 & 3.62 \\
\hline For s & & & & & & \\
\hline VF & 6 & 11.9 & 20 & 32.93 & 1.502 & 2.89 \\
\hline VT & 6 & 11.9 & 20 & 34.14 & 1.588 & 2.38 \\
\hline VW & 6 & 10.53 & 20 & 29.83 & 1.378 & 2.27 \\
\hline For s & & & & & & \\
\hline ZF & 6 & 26.53 & 20 & 72.57 & 3.288 & 6.81 \\
\hline ZT & 6 & 23.09 & 20 & 64.73 & 2.974 & 5.27 \\
\hline ZW & 6 & 22.44 & 20 & 60.26 & 2.701 & 5.98 \\
\hline
\end{tabular}


Dependencies for saccade movements, received by the adopted method for 2 observers $M$ and $Z$ with a sampling frequency of $500 \mathrm{~Hz}$ and for the observer $V$ with a high sampling speed of $1250 \mathrm{~Hz}$ also for saccade movement are shown in Fig. 6. Data were obtained when averaging on turns by an angle of an increment $45^{\circ}$ for 3 images, as usual designated by the letters $F, T, W$.

It is possible to note that the measurements, given on Fig. 6, by means of a proposed method for processing of saccade movements, show that all dependences for slow samplings $(500 \mathrm{~Hz}$, observers $M$ and $Z$ ) are located higher than the corresponding dependences for the observer $V$, for whom the data were collected with a high frequency of $1250 \mathrm{~Hz}$. For observers with large time intervals between samplings, a stronger divergence of linear dependencies than for the observer $\mathrm{V}$ with a higher portion of the sample $(1250 \mathrm{~Hz})$ is observed. It is probably due to the higher speed and, accordingly, the divergence in the saccade movement between large time intervals, characteristic for more rare samplings.

Data in Fig. 7 shows that there is a noticeable difference in the calculated dependences for fixations for all types of images $(F, T W)$ for the observers $M$ and $Z$ (for which the sampling was carried out at a frequency of $500 \mathrm{~Hz}$ ). It is possible to note that the observer $V$ has a nature of linear dependences that is very close for all 3 types of the used images that, perhaps, testifies about features of a visual apparatus of this observer. This observer also has a more noticeable and quick out to a linear dependence by size of the window $Z$ in comparison with the more gradual out of the observers $M$ and $Z$.

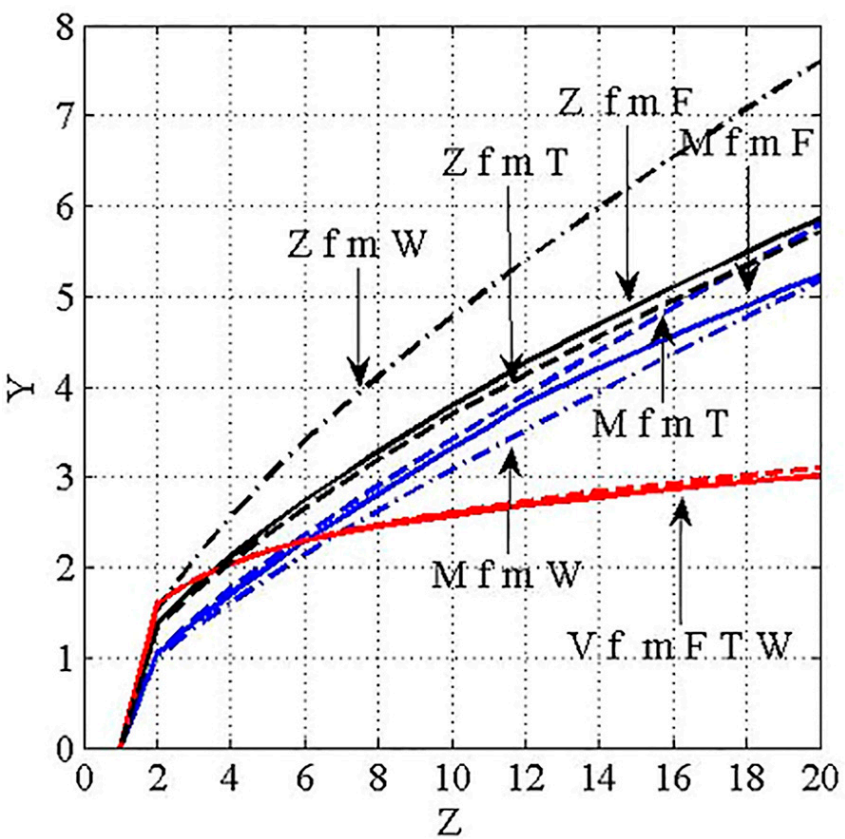

Fig. 7. Dependence for fixation movements for observers $M, Z$ and $V$.

Таблича 6 крис. 7.

\begin{tabular}{|l|l|l|l|l|l|l|}
\hline For s & $\mathrm{X} 1$ & $\mathrm{Y} 1$ & $\mathrm{X} 2$ & $\mathrm{Y} 2$ & $\mathrm{~b}$ & $\mathrm{a}$ \\
\hline MF & 8 & 2.9 & 20 & 5.225 & 0.194 & 1.345 \\
\hline MT & 8 & 2.9 & 20 & 5.86 & 0.246 & 0.94 \\
\hline MW & 8 & 2.6 & 20 & 5.166 & 0.213 & 0.90 \\
\hline For s & & & & & & \\
\hline ZF & 8 & 3.282 & 20 & 5.86 & 0.215 & 1.56 \\
\hline ZT & 8 & 3.191 & 20 & 5.706 & 0.21 & 1.506 \\
\hline ZW & 8 & 4.11 & 20 & 7.605 & 0.291 & 1.782 \\
\hline For s & & & & & & \\
\hline VF & 8 & 2.453 & 20 & 3.013 & 0.046 & 2.093 \\
\hline VT & 8 & 2.62 & 20 & 3.096 & 0.040 & 2.232 \\
\hline VW & 8 & 2.44 & 20 & 3.005 & 0.047 & 2.065 \\
\hline
\end{tabular}

\section{CONCLUSION}

The paper presents an analysis for trajectory pattern of an observer's eye movement on a screen with flat image, based on a separate consideration of saccade and fixation eye movements using sliding averaging window for realization to calculate a root-meansquare deviation in the $X$ and $Y$ coordinates of realizations.

It was found that the corresponding approximate linear estimates for change of the sliding average window of averaging for saccade movements really form a set of curves, gradually diverging with increasing size of a dependence window. 
Assessment for eye movements in fixation areas according to an image on a screen, calculated using the same method of a sliding window, has a slightly different character. The corresponding dependences quickly come to areas with a linear dependence $y=a+b x$, but with a notable difference for different observers by parameter of the coefficient $a$. Comparison for realizations, received with use of normal selection rate of $1250 \mathrm{~Hz}$ and much slower selection of $500 \mathrm{~Hz}$, clearly showed that in the latter case, when sharp increases in intervals between selections take place, a growth of movement speed in saccades starts being shown and linearity, which was well visible for selections with a frequency of $1250 \mathrm{~Hz}$, is broken.

It is shown that the used approach can be applied in tasks to distinct the observers when viewing identical images.

The work is performed with assistance of the RFBR (grant No. 13-07-00834).

\section{REFERENCES}

1. Belyaev RV, Kolesov VV, Men'shikova GYa, Popov AM, Ryabenkov VI. Issledovanie osobennostey vospriyatiya videoinformatsii pri pomoschi fraktal'nogo analiza traektorii dvizheniya glaz [Study of Special Features of the Perception of Video Information by the Fractal Analysis of the Trajectory on the Eyes Motion]. RENSIT, 2011, 3(1):56-68.

2. Barabanschikov VA, Zhegallo AV. Metody registratsii dvizheniy glaz $\mathrm{v}$ psikhologii: oanovy uchebno-metodicheskogo kompleksa [Methods of recording eye movements in psychology: foundations of educational and methodical complex]. Eksperimental'naya psikhologiya, 2014, 7(1):132-137.

3. BelyaevRV,KolesovVV,Men'shikovaGYa,
Popov AM, Ryabenkov BI. Issledovanie individual'nykh osobennostey dvozheniya glaz na osnove tekhnologii Eye Tracking [Study of the individual characteristics of eye movements on basis of technology Eye Tracking]. Proc. XVIII international scientific-practical conference "Fundamental and applied research, development and application of high technologies in industry and economy", 5-6 dec. 2014, St. Petersburg, Russia, pp. 1218.

4. Belyaev RV, Kolesov VV, Men'shikova GYa, Popov AM, Ryabenkov VI. Analiz vidov dvizheniya glaz s pomosch'yu fraktal'nykh algoritmov [Analysis of the Types of the Eyes Movement by Fractal Algorithm]. RENSIT, 2014, 6(1):30-43. 